\title{
10-Year Outcomes of the Charnley and Exeter Total Hip Replacements: A Clinical Cohort Study Conducted in Cappagh National Orthopaedic Hospital (CNOH) Dublin/Ireland
}

\author{
Mohamed Monawar, MBBS, MRCS, MCh, Orth ${ }^{1 *}$, Paddy Kenny, MB, BCH, BAO, MFSEM, FRCSI, FRCS \\ (Tr \& Orth) ${ }^{2}$, Natasha Rafter, RCSI ${ }^{3}$, Fionnuala Walsh ${ }^{4}$ and Samir Talha, MBBS, MRCS ${ }^{5}$
}

${ }^{1}$ Trauma \& Orthopaedics Registrar, Cappagh Hospital, Ireland

${ }^{2}$ Orthopaedic Surgeon - Cappagh Hospital, Ireland

${ }^{3}$ Department of Epidemiology and Public Health Medicine, Royal College of Surgeons Ireland (RCSI), Ireland

${ }^{4}$ Clinical Nurse Manager (CNM), Cappagh Hospital, Ireland

${ }^{5}$ Trauma \& Orthopaedic registrar, Cappagh Hospital, Ireland

*Corresponding author: Mohamed Monawar, MBBS, MRCS, MCh Orth, Trauma \& Orthopaedics Registrar, Cappagh Hospital, Dublin, Ireland

\begin{abstract}
Background: Cappagh National Orthopaedic Hospital $(\mathrm{CNOH})$ joint register is of huge value in assessing current outcomes of Charnley and Exeter Total Hip Replacements (THR) in Ireland. It is the chief orthopaedic elective hospital and as the Republic of Ireland has no published results from the National Joint Register yet; focusing on this register will help the Irish orthopaedic community by demonstrating which of the two devices has superior survivorship and patient reported outcomes.
\end{abstract}

Methods: Joint register data for all patients who underwent the Charnley and Exeter THR between 2005 and 2006 was reviewed in 2017. Information regarding demographics, indication for surgery, quality of life questionnaires (WOMAC) scores and revision rates was assessed, and survival statistics was used to determine survivorship.

Results: A total of 348 Charnley and 232 Exeter THRs were implanted between 2005 and 2006 with an average followup of 10 years. Charnley THRs had a mean age of 68 years and BMI of 29.1 while the Exeter group had a mean age of 66.7 years and BMI of 28.7 at the time of surgery. Primary osteoarthritis was the surgical indication in more than $90 \%$ of both groups.

The Charnley group had seven revisions $(97.9 \%$ overall survivorship) and the Exeter group had two revivions (99.1\% overall survivorship) by 2017 . The WOMAC scores in the Charnley group reduced from 60 to 24 while in the Exeter group reduced from 58 to 20 ( $p=0.08$ Mann-Whitney $U$ ).
Conclusion: Our results from $\mathrm{CNOH}$ are excellent in terms of survivorship and consistent with international figures, we would advice to continue this cohort for a longer review to assess the outcomes at 15 and 20 years.

\section{Introduction}

\section{Osteoarthritis}

Osteoarthritis $(O A)$ is defined as a clinical syndrome of joint pain with variable levels of reduced joint function and quality of life, $O A$ is the most common form of arthritis [1]. The features of OA include areas of articular cartilage loss within synovial joints, hypertrophy of bone which results in osteophyte formation and subchondral bone sclerosis, and capsule thickening, the clinical condition is characterized by arthralgia and tenderness, limited joint motion, crepitus, effusions, and occasional local inflammation [2]. Any joint can become a target of OA but is most common in the knee, hip, hand, foot and spine [2]. OA affects an estimated $10 \%$ of men and $18 \%$ of women over 60 years of age worldwide [3]. The condition affects 400,000 people in Ireland and there has been an increase in the number of people with OA in Ireland for two reasons; the ageing population and obesity (the additional weight causes an increased strain on the joints) [4]. The prevalence of OA in Ireland 
is $12.9 \%$ in people aged over 50 years based on a selfreported definition (when people were asked 'do you have osteoarthritis'?) with a prevalence rate of $9.4 \%$ for men and $17.3 \%$ for women [5].

\section{Hip osteoarthritis}

In the lower limb, the hip is the second most common joint affected by OA preceded by the knee [6]. Symptomatic OA of the hip causes immense pain, reduced quality of life and functional disability [7]. The aetiology of hip OA is influenced by many factors such as genetics, biomechanics, systemic and biochemical stimuli [8]. Risk factors associated with hip OA are age, history of trauma, obesity, heavy physical labour, longterm farming $>10$ years, certain joint loading sports and congenital conditions [9]. Diagnosis of primary OA of the hip is based on patient symptoms, thorough clinical examination to exclude other causes and radiography. Management of hip OA is approached in a systematic way, firstly through conservative therapy with activity modification and analgesia, followed by a course of physiotherapy, ambulatory aids to offload joint strain and weight loss [10]. Intra-articular steroid injections of the hip are very helpful as they can alleviate pain and also differentiate hip OA pain from referred sources such as the back or the sacroiliac joints [11]. Once conservative therapy has failed or become ineffective due to end stage disease then proceeding to surgical intervention by joint replacement is indicated.

Other causes for surgical management include inflammatory arthritis, fracture, developmental dysplasia of the hip (DDH), Avascular necrosis (AVN), infection and malignancy [12].

\section{Total hip replacement}

Total hip replacement (THR) is usually the final option once you have exhausted other treatment modalities as it is a serious and permanent procedure. The ideal candidate for THR is a patients who experiences severe hip pain most of the time in addition to reduced activities of daily living, and on obtaining radiographs of the pelvis, extensive narrowing of the joint spaces are observed [13]. THR can be a costly choice however when comparing cost effective analyses of expenses incurred from using medication long-term and lost productivity, the price of surgery in patients with severe symptoms is resourceful [14].

The indications for THR in the United Kingdom (UK) are OA (93\%), osteonecrosis (2\%), femoral neck fracture $(2 \%)$, developmental dysplasia of the hip (2\%), and inflammatory arthritis (1\%) [15].

The hip joint is a very important structure as it allows us to walk, run and jump while supporting our body weight. It is described as a synovial ball and socket joint, the articulation of the ball shaped femoral head with the socket-shaped acetabulum provides stability which is enhanced by the joint capsule, ligaments and large muscle groups [16].

THR is performed by removal of the femoral neck
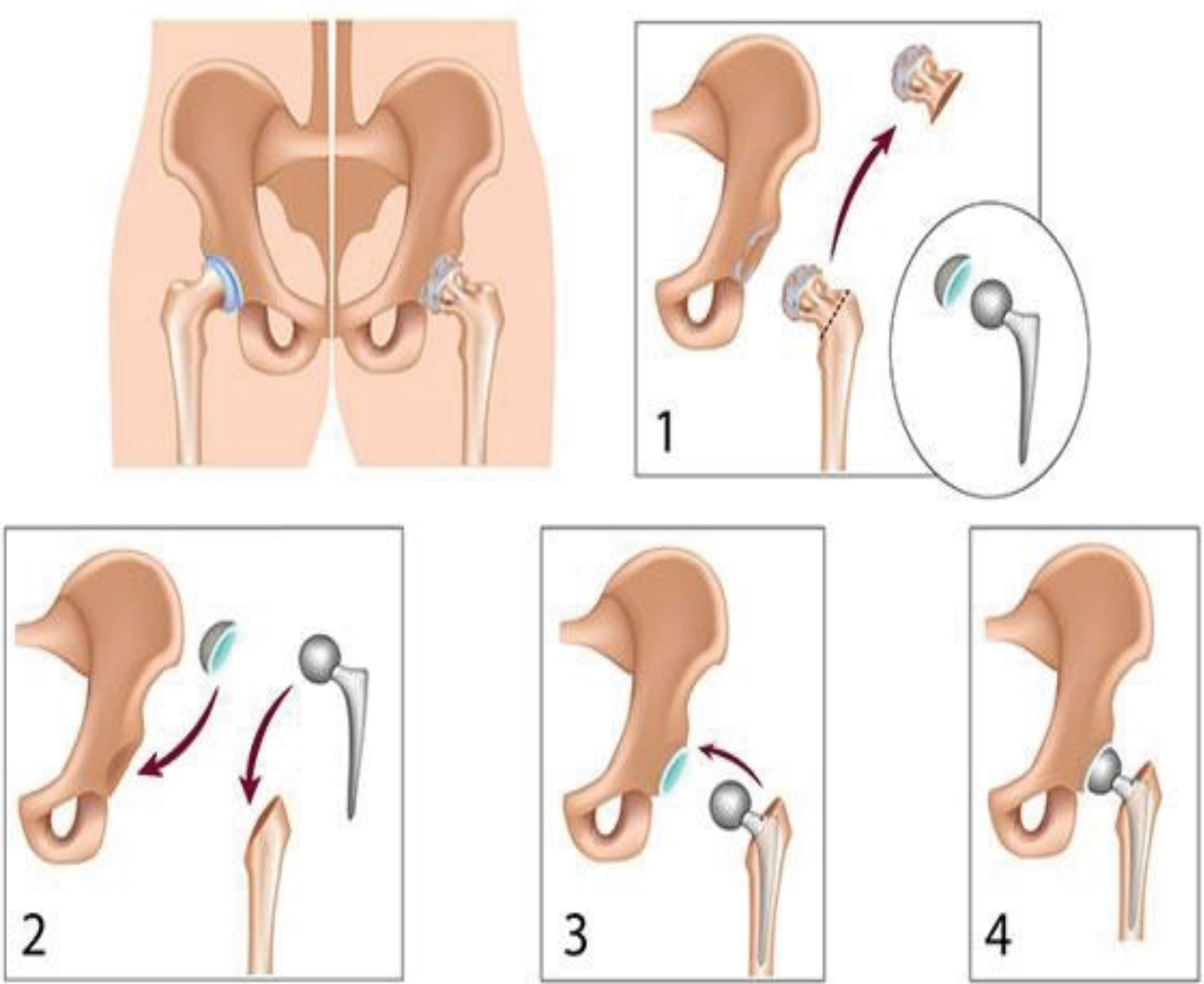

Figure 1: Diagram showing the stages of a Total Hip Replacement. 
by resecting it just below the femoral head as well as taking away the acetabular cartilage through successive reaming (Figure 1). The second step is to prepare the femoral medullary canal using broaches, cement is applied and then the femoral stem and acetabular cup are implanted [16]. The surgical goal of a THR is to restore the anatomy and biomechanics of the hip joint to a pre-disease level as best as possible. To achieve a successful THR patients should have improved hip abductors and joint mobility post-operatively, and the surgeon should aim at to minimise the post-operative complications which could range from a limp to implant failure [17].

THR is an effective intervention for hip OA. In the United States (US) more than 300000 THRs were performed in 2010, in the United Kingdom (UK) about 80000 are performed yearly and at least 4500 THRs are undertaken annually in Ireland [18,19].

\section{Types of Total Hip Replacement (Cemented - Uncemented - Hybrid)}

Cemented total hip replacement: Cemented THR was first performed by Glück in 1891, his approach was to use bone cement to improve implant fixation and bonding, however it was Sir John Charnley in the late 1950's that spread this technique by utilising a cement that is used in dental surgery [20].

Polymethylmethacrylate (PMMA) is the substance which is frequently used for implant fixation due to the notion that cement interdigitates within bone. Cement performs as a grout between the stem and bone, hence there is no attachment between them. During the early period of cementing certain factors play a useful role in altering regular bone function which aids in fixation and bonding such as; thermal, chemical, mechanical and vascular trauma which damages the endosteal blood supply resulting in necrosis which can reach a depth of 500 micrometres. After a few months the endosteal blood supply is restored as fibrovascular granulation tissue creating a fresh interface between the cement and bone [21]. The risk of revision published in a report by the National Joint Register in the UK estimates that at 12 years after THRs the lowest rates were in the cemented fixation population at $3.93 \%$ compared to $5.46 \%$ in the uncemented group [22]. Cemented THR remains the gold standard in terms of long-term clinical results [23].

Uncemented total hip replacement: Uncemented THRs unlike cemented have a special coating called hydroxyapatite which permits on-growth of bone and thereby fixation of the implant [24]. It is assumed that by this method a that true biologic bond occurs during implantation between the stem and bone as the coated surface of the metal implant encourages the on-growth of bone onto the prosthesis [21]. Uncemented THR achieve stability and fixation by sustaining a strong interface between bone and implant aided by bony intercalation onto the implant due to bone remodelling phenomena which occurs after disruption of normal bone function [25].

Hybrid total hip replacement: A Hybrid THR prosthesis is comprised of a cemented femoral stem and an uncemented acetabular cup. This is a good option for young, active patients as it preserves bone, aids in revision and provides a solid fixation [24]. A major study in Norway showed that the use of hybrid THRs offers better survivorship than a cemented socket in the younger patient [26].

Charnley Total hip replacement: The Charnley stem (Depuy Synthes; Johnson \& Johnson) named after Sir John Charnley who is regarded as the pioneer of successful cemented THR is described as a low friction arthroplasty of a monoblock design with a collared stem and, it is considered the gold standard for cemented hip replacement [27]. This collar has three main functions as noted by Sherfey, et al.: As an aid in cement pressurisation, to align the stem within the cement mantle, and to evenly distribute load to the proximal femur [28]. It has an Orthopaedic Data Evaluation Panel rating (ODEP) of 10A indicating that the Charnley prosthesis has a minimum 10 year follow up with strong evidence of its use [29].

The Charnley stem is occasionally criticised due to its monoblock design when compared to the modular systems as it can lack flexibility which results in difficulty in changing the leg length and offset. To address these difficult factors surgeons rely on preoperative radiographic templating and planning, meticulous intraoperative cementing technique as well as optimisation of abductor tension to ensure stability [30].

A review by Poon, et al. in Middlemore Hospital showed that the Charnley stem has a $95.6 \%$ survivorship at 7 years [31]. Long term results at a minimum of 20 years estimated a $5 \%$ revision rate for aseptic loosening using the Charnley stem after improved cementing techniques were used [32]. Other studies had showed up to a $16 \%$ risk of revision for aseptic loosening of the Charnley stem at 20 years [33]. A follow up report completed by Mullins, et al. revealed a 10-year survival of $93 \%$ deteriorating to a 30 -year survival of $73 \%$ of Charnley THRS which were performed through the posterior approach [34]. During a Charnley meeting in Lyon - France, Caton, et al. published a survival curve of $85 \%$ after 25 years showing excellent long-term results [35]. Berry, et al. reported similar results with $86.5 \%$ survivorship for aseptic loosening at 25 years and also noted that men had a twofold higher rate of revision for aseptic loosening than women [36]. Results from the Norwegian Arthroplasty Register showed that the Charnley prosthesis survivorship improved markedly over time, and beyond 18 years had better survival than 


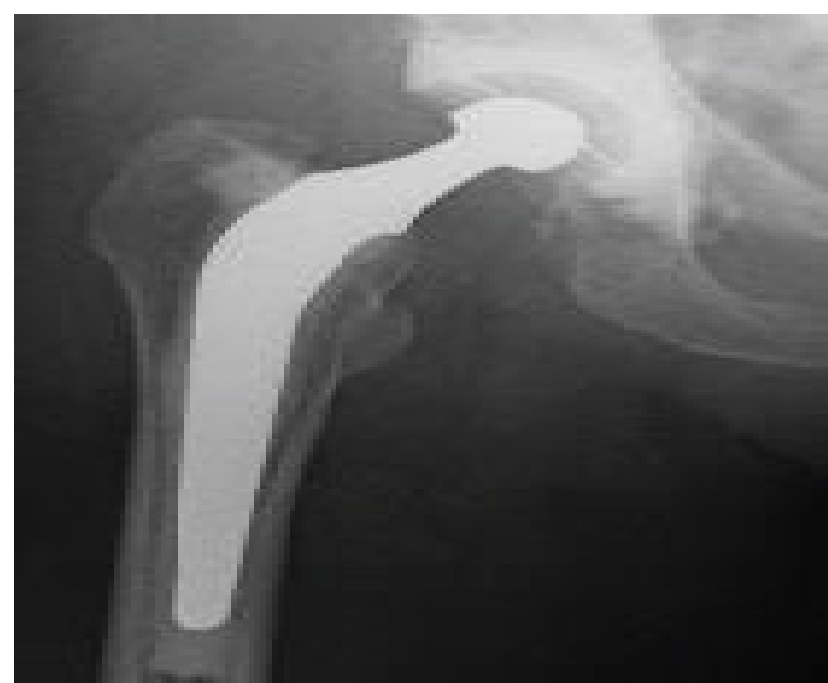

Figure 2: Radiograph of the Charnley THR.

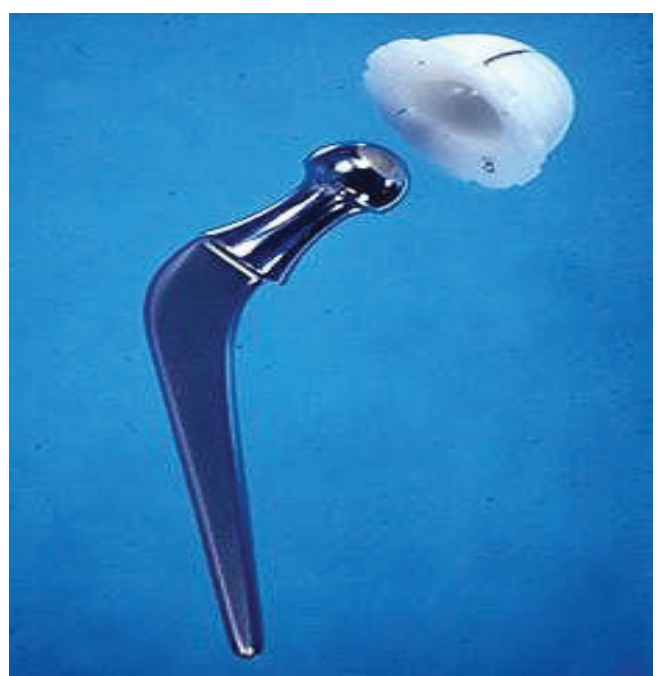

Figure 3: The classical Charnley THR (note the stem collar).

other cemented THRs [37]. The Australian National Joint Registry's Annual report for 2016 revealed a revision rate of $8.2 \%$ at 10 years for the Charnley Hip Replacement while less revision rates of $5.9 \%$ at 10 years had been seen in the Nordic Arthroplasty Register [38,39] (Figure 2 and Figure 3).

Exeter total hip replacement: The Exeter stem (How medica Osteonics; Stryker) introduced in 1970s by Ling and Lee has been through minimal changes since its first use, in 1988 the surface finish of the stem became polished and the stem design was converted to a modular form known as the Exeter Universal stem [40]. This modularity at the head neck junction allows for adjustment of leg length, offset and hip version which can differ greatly between patients [30].

The Exeter Universal stem is a collarless, double tapered, highly polished wedge, with essentially a rectangular cross section throughout [28]. Williams, et al. described the rationale of this design; as the stem is loaded, the polished taper slightly subsides within the cement mantle. This increases hoop stress within the mantle and the cement responds with a degree of creep. Therefore, the shear stress is converted to radial compressive stress and transmitted to the cement bone interface thus decreasing the shear stress at this interface during load transmission. This allows the stem to fit tighter with load and protects the cement mantle by producing compressive forces thereby continually tightening throughout the life of the hip $[28,40]$. It also has an ODEP rating of $10 \mathrm{~A}$.

Out of 38,381 cemented Total Hip Replacements performed in the UK in 2012 66\% used an Exeter stem [41]. In a prospective study comparing both the cemented Exeter stem and the Endurance stem (collared and trapezoidal in shape proximally, circular in cross section distally) over a minimum of seven years showed the Exeter patients had no revisions for aseptic loosening of the femoral stem while the Endurance patients had 5 revisions and 2 impending revisions for aseptic loosening (20.5\%) of the femoral stem [28]. In Iceland a retrospective review of 654 primary Total Hip Replacements using the Exeter stem showed a 10 -year revision rate of $4 \%$ using aseptic loosening as an endpoint and $7 \%$ using all revisions as an endpoint [42]. Similarly a study performed by Carrington, et al. on an update of their previous report now at 17 years reviewed the survivorship of Exeter stems and when taking aseptic loosening of the stem as the endpoint was $100 \%$ [43]. Other reports have also shown excellent long-term results of the Exeter THR with survivorship of $99 \%$ at 22.8 years with an endpoint of revision for aseptic loosening or lysis of the stem, and when considering revision for all causes including infection and periprosthetic fracture, survivorship was $95.6 \%$. The overall survivorship of the Exeter THRs in the same study when taking into account revision of either components for any reason as an endpoint at 22.8 years was $82.9 \%$ [44]. The Exeter THR has similarly shown excellent results in the under 40's age group where Kaplan-Meier survival analysis displayed a probability of survival of the femoral component of $95.8 \%$ with revision for any reason as the endpoint; $100 \%$ for aseptic loosening, and $96.7 \%$ for radiological failure [45]. In a ten-year stem analysis from the Norwegian Arthroplasty Register the Exeter stem produced the best survivorship results when compared to the Charnley and other cemented THRs, however the Charnley stem surpassed the Exeter and other cemented stems at 18 years [37]. The National Joint Registry in the UK shows that for the Exeter stem produced a 10-year revision estimate of $2.23 \%$ [22]. However, in the Nordic Arthroplasty Register the overall implant survival of Exeter Hip Replacements was 93.5\% [38] (Figure 4 and Figure 5).

Revision Total Hip replacement: Revision THR is a surgical intervention performed for failed Primary THR most commonly due to aseptic loosening, infection, recurrent dislocation and periprosthetic 


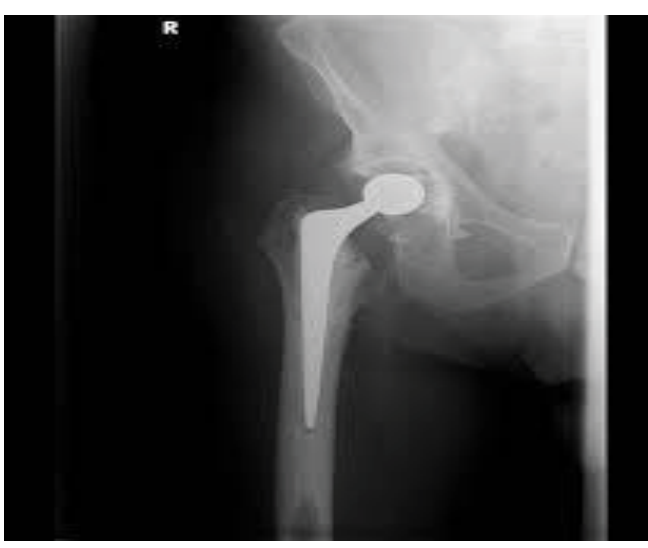

Figure 4: Radiograph of the Exeter THR.

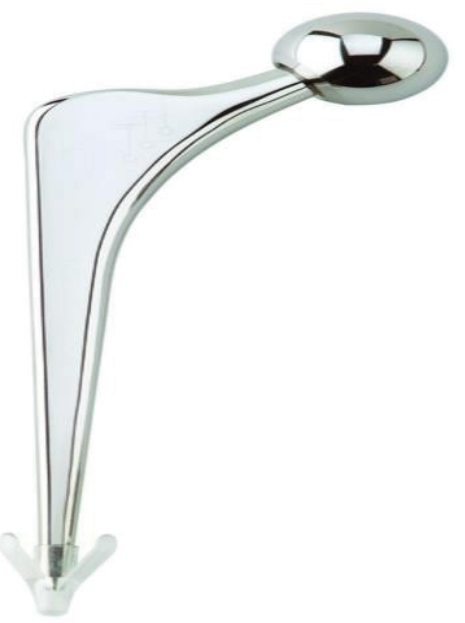

Figure 5: Image showing the Exeter Universal Stem.

fractures [46]. Revision surgery results in higher costs to health providers, operations take a longer time, they necessitate a lengthy hospital stay for recovery and implants are more expensive when compared to Primary THR [16]. Complication rates are all higher with revision surgery than with primary THR and infections, dislocations and mortality can pose a high risk [16]. In the US, more than 50,000 revision THRs are performed annually with costs exceeding a staggering $\$ 1$ billion [47]. In the UK ten percent of all THRs are revisions and $55 \%$ of the revisions are due to aseptic loosening $[46,48]$. The National Institute for Health and Care Excellence (NICE) previously suggested a tenyear revision rate of $<=10 \%$ or less as an acceptable benchmark performance of a primary total hip implant [49]. However, NICE has recently revised the benchmark at 10 years and recommends it is reduced from $10 \%$ to 5\% [19]. Therefore, it is important that Primary THRs are performed in the best possible manner using an excellent prosthesis with proven results to reduce the risk or delay the need for Revision hip surgery.

\section{Joint registries}

Joint registries are used to collect and store information on joint replacement procedures, to monitor the performance of joint replacement implants and the effectiveness of different types of surgery, and to improve clinical standards to benefit patients, clinicians and the orthopaedic sector as a whole [50].

In the past orthopaedic surgeons frequently depended on small cohort analyses to assess outcomes and complications. Currently, joint registries at hospital based level and national levels have been implemented including the more recent American Joint Replacement Registry (AJRR) to provide an invaluable source of information for orthopaedic surgeons, patients, healthcare administrators, regulators, and implant suppliers [51]. The goals of a National Joint Registry as outlined by Maloney, et al. are: (A) To precisely define the epidemiology of joint replacement surgery in a patient population; (B) To deliver appropriate evidence to the orthopaedic community on the outcomes of joint replacement; (C) To reveal risk factors for a poor outcome, and to improve that outcome through continuous feedback to participating centers and surgeons [52].

Joint registries allow for the observation and follow up of new implants and designs by identifying potential problems and complications. The National Joint Registry of England and Wales detected that metalon-metal stemmed articulations led to high rates of implant failure and revisions compared to other bearing surfaces, mostly in young women implanted with large diameter femoral heads, it recommended to avoid their use and to monitor patients who have them implanted [53].

Sweden demonstrated that by establishing its National Joint Register in 1979 it was able to drive down the revision burden over subsequent decades; the burden of revision was $6.4 \%$ in Sweden versus $16.9 \%$ in the US which had no registry at the time [54]. The most important factor found to reduce the risk of revision due to aseptic loosening was good surgical technique however the implant used also played an important role [55].

Several countries have established an arthroplasty register, The Swedish National Arthroplasty Registry being one of the earliest. Other countries that have a register include Finland, Norway, England/Wales, Australia, Denmark, New Zealand, Hungary, Canada, USA, Scotland, Romania, South Africa and Malawi. All registries except for the England and Wales National Joint Register are managed by Orthopaedic surgeons and without any government control [56].

Ireland has recently established a National Joint Register which is located in the South Infirmary-Victoria Hospital (SIVUH) in Cork Under the Governance Board of the National Office of Clinical Audit (NOCA). NOCA is responsible for the administration of the Governance Committee which has representatives from Irish Institute of Trauma and Orthopaedic Surgery (IITOS), Irish College 
of General Practitioners (ICGP), HSE Quality and Patient Safety Directorate, Arthroplasty/Orthopaedic Nursing Specialists and many more.

The Irish National Orthopaedic Register (INOR) went live in SIVUH since May 2016 and since early 2017 in Midland Regional Hospital, Tullamore. An expansion plan for INOR implementation in the other remaining 27 elective orthopaedic Statutory and Independent Hospitals (including Cappagh) in Ireland is under review $[57,58]$.

Cappagh national orthopaedic hospital - Joint register: Cappagh National Orthopaedic Hospital ( $\mathrm{CNOH}$ ) is Ireland's chief hospital for elective orthopaedic surgery, it caters for both public and private patients with a 159-bed capacity. $\mathrm{CNOH}$ is the largest dedicated orthopaedic hospital in Ireland and located in the capital, Dublin. There were 22 Consultant Orthopaedic Surgeons appointed to the hospital at the time of this study, all of whom share contracts with 7 acute Dublin hospitals. Around 600 hip replacements are performed yearly in Cappagh Hospital [59].

The first hospital based joint register in Ireland and was established in Cappagh Hospital in 2005 by Mr. P. Kenny (Consultant Orthopaedic Surgeon). The register began as a pilot study, led by a project team comprised of one Consultant Surgeon, the Clinical Nurse Manager (CNM) involved in research and audit, the IT manager and an orthopaedic NCHD (Non-consultant hospital doctor). The initial study included all primary and revision hip and knee replacements performed by 5 participating orthopaedic surgeons. Following the success of the pilot study the project then expanded in January 2006 to include all surgeons who perform joint replacement surgery at $\mathrm{CNOH}$.

The current structure of Cappagh joint register is that it is managed by a clinical nurse manager (CNM) as well as a part time nurse who review patients, arrange radiographs (Anteroposterior ' $A \mathrm{P}^{\prime}$ ' pelvis for hips - AP + Lateral weight bearing for knees), and fill in an online questionnaire detailing any complications (revision, infection, dislocation, periprosthetic fracture, limb length discrepancy) since the time of surgery. An orthopaedic registrar is also appointed to the joint register and is called upon if any abnormalities are noted on the radiograph reports or if the clinical situation of the patient requires a review.

Patients fill out a self-reported quality of life questionnaire (at each review 6 month - 5 years - 10 years) 'The Western Ontario and McMaster Universities Arthritis Index' (WOMAC) Appendix 1 [60]. The results of the WOMAC scores and the online complications questionnaire are recorded on a joint register data sheet which is managed and updated by the Joint Register data controller (CNM). The hospital's IT manager is also involved in managing and upgrading the Joint Register data base.
From January 2005 to December 2016 there have been 13,034 primary joint replacements $(7,526$ hips and 5,381 knees) and 1,682 Revision joint replacements (1,294 hips and 388 knees) performed in $\mathrm{CNOH}$ and recorded in Cappagh Hospital's Joint Registry.

\section{Aims and Objectives}

The aim of this study is to review the long-term results of Hip Replacements performed at Cappagh National Orthopaedic Hospital.

I. Primary outcome is to compare the survivorship (time period from primary surgery to revision surgery) and the revision rates of the two frequently used cemented hip prosthesis (Charnley/Exeter) at 10 years follow up.

II. Secondary outcome is to compare the complication rates (Infection/dislocation/ periprosthetic fractures) \& assess the Patient Reported Outcome Scores (PROS) at 10 years follow up of the Charnley and Exeter Hip Replacements.

We used the PICO principle which is a technique used to frame and answer a clinical or health care related question;

$\mathrm{P}=$ Population/problem/patient

$\mathrm{I}=$ Intervention

$\mathrm{C}=$ Comparison/controll

$\mathrm{O}=$ Outcome

- P- All patients attending Cappagh Hospital for elective primary (initial surgery to hip joint) Total Hip Replacement between January 2005 and December 2006.

- Cemented Hip Arthroplasty.

- C- Compare Charnley Hip Replacement with Exeter Hip Replacement.

- Survivorship of each implant taking Hip Replacement Revision surgey for aseptic loosening as the end point.

\section{Methodology}

\section{Study design}

A clinical cohort study examining the long-term outcomes (10-12 years post-surgery) of elective Primary THRs survivorship of the Charnley and Exeter prosthesis.

\section{Setting}

The study was performed in Cappagh National Orthopaedic Hospital using the Cappagh Joint Register.

\section{Study population}

The sampling frame was patients with Charnley or Exeter THRs who had their primary hip or knee 
replacement at Cappagh Hospital between 2005 to 2006 and were recorded in the Cappagh Joint Register.

\section{Participants}

Patients with Charnley and Exeter THRs.

\section{Inclusion criteria}

- Charnley or Exeter THRs at Cappagh National Orthopaedic Hospital between 2005 and 2006 which were recorded in the Cappagh joint register.

\section{Exclusion criteria}

- Patients who underwent other types of THRs

- Total Knee Replacements (TKRs)

- Hip Resurfacing Procedures

- Patients who we could not contact

- Patients who opted out of the joint register

\section{Outcomes}

\section{Primary outcomes}

Survivorship of Charnley and Exeter THRs taking revision surgery for aseptic loosening as the end point.

\section{Secondary outcomes}

- Complication rates (Infections, dislocations and periprosthetic fractures)

- Patients reported outcomes through the WOMAC scores.

\section{Data collection}

Data was collected within the existing clinical review framework at $\mathrm{CNOH}$. As part of this, all patients who have had a primary or revision TKR or THR are reviewed in $\mathrm{CNOH}$ at intervals of 6 months, 2 years (telephone interview) and every following 5 years in the outpatient department. Review is life-long or until revision is required, in which case review will be every 2 years.

Baseline data was collected pre-operatively (age/ gender/height/weight/body mass index (BMI)/medical conditions) at the pre-assessment clinic (PAC) which is usually held a few weeks prior to the date of surgery and also on the day of surgery by the ward nurses. The data is recorded electronically and written in the patients chart.

At the time of surgery implant details contained in the manufacturers label are recorded onto $\mathrm{CNOH}$ IT system known as CAPAS. All implants used at $\mathrm{CNOH}$ contain this data in a barcode which is placed into the operative notes section in the patients handheld medical chart and also uploaded onto CAPAS using a barcode scanner by the operating theatre nurses.

Data in relation to anaesthetic, surgical technique, implantsused, antibiotictherapyand thromboprophylaxis are recorded electronically onto a medical information system called Bluspeir by the surgical team. The Bluspeir system integrates with CAPAS system and there by forms as a feedback mechanism in case errors occour in recording the patients implant details.

The Joint register system is an electronic component of Bluspeir and data recorded at the time of operation triggers the generation of the post-operative joint register review date. Letters are then posted by a medical secretary to the patients notifying them of their review date in $\mathrm{CNOH}$ joint register. Patients however are seen much earlier at the performing surgeons outpatient's department clinics at six weeks after surgery to ensure a successful early post operative outcome.

At each review post-discharge complications are documented in the joint register system so that an electronic record of adverse outcomes is available. The joint register questionnaire is a standard form on the Bluspeirs IT system which evaluates if patients have had any major complication since the time of surgery or since the time of last review. It is checked in every review clinic. It measures whether patients had revision surgery, THR infections, periprosthetic fractures of the hip, dislocations of the hip and any leg length discrepancy noted after surgery (Appendix 2).

The WOMAC form, a self-administered quality of life questionnaire is used to ascertain the patient's perception of their pain, function, disability and emotional status concerning their arthritis. This is a widely-used questionnaire by health professionals to evaluate the condition of patients with osteoarthritis of the knee and hip, including pain, stiffness, and physical functioning of the joints. The WOMAC forms are completed ideally by the by the patients themselves however if that is not possible due to any reason then whoever is accompanying the patient may assist in filling out the form, at times it may be required for the person leading the joint clinic review to assist the patients with the form if they are alone and not able to manage. The WOMAC questionnaire is then handed to an outpatient staff member (medical secretary) who is not in clinical contact with the patients to reduce the chances of influencing the answers and a score is calculated from the form, the lower the score the better the outcome with an overall score of 96 (Appendix 1).

$A$ radiograph is also performed in the radiology department of $\mathrm{CNOH}$ of the corresponding joint (Anteroposterior 'AP' Pelvis for THRs - AP + Lateral views for TKRs). The radiographs are then reported by two senior radiologists in $\mathrm{CNOH}$, however these reports are not linked to Bluespeir system and need to be seen in a separate electronic radiograph viewing system. To obtain the study cohort all patients who had hip or knee replacements were called for their standard 10 year review. The subset who had Charnley or Exeter THRs were then selected from the reviewed patients. 
Data for the study was collected at designated physician and nurse-led clinics which were held three times a week (Monday to Wednesday) at morning and afternoon sessions between the periods of November 2016 to May 2017.

In line with standard practice all patients who do not attend (DNA) the review clinics are contacted by telephone and the review is done remotely, necessitating attendance for radiograph at a local imaging centre. Patients who DNA twice and are difficult to trace are actively persued through their Surgeons or GP. Patients who DNA three times are removed from the joint register review clinics. Patients who are in long term care are not called to the joint register review clinics, however information about revision surgery and whether they have had any complications are taken from their care provider (nurse) by telephone. Data on patients who have passed away regarding revision or complications with the THR are recorded on the joint register database through information obtained from either the next of kin or GP.

\section{Data protection}

Anonymous patient data was stored separately and encrypted from the raw data on a password protected computer in Cappagh outpatient's department. Patients' written consent was taken pre-operatively to participate in the Joint Register and to use their data for research (Appendix 3).

\section{Ethics}

The joint register has approval to conduct research on data collected (Appendix 4). Approval from the head surgeon leading the Joint Register and nurse in charge of the Joint Register (data controller/CNM) was sought prior to undertaking this study. No medical records outside of the joint registry data were used for this study and additional review by a research ethics committee was not required.

\section{Study size}

To achieve an ideal study size for the purpose of this research we would need to incorporate multiple institutions or provide a multicentre study. Due to time constrictions and feasibility we considered to focus on our local cohort at $\mathrm{CNOH}$ thereby taking all patients who underwent a primary THR with the Charnley and Exeter prosthesis.

\section{Statistical methods}

Data was analysed with IBM SPSS Statistics software for Windows version 24.0 (IBM Corp., Armonk, N.Y., USA).

Demographic data was analysed using descriptive statistics to calculate the mean age at time of surgery for each implant and per gender. We also compared patients mean ages, male and female distributions, and patients BMI scores.
Patient reported outcomes were compared using the Mann-Whitney $U$ test to compare differences between the Charnley and Exeter WOMAC scores at ten years post-surgery.

Regression analysis was performed to assess for any correlation between ages of the patients and stem type used. Chi-Square test was used to determine any significance between sex and stem design implanted.

Kaplan meier survival analysis was used to assess survivorship of the femoral stems taking revision for aseptic loosening as the end point primarily, and for survivorship of the femoral stems taking revision for other causes as the end point thereafter. The Log Rank test was used to measure for equality of survival distributions between the stems.

\section{Results}

Between January 2005 and December 2006 '1,433' primary THRs had been performed in $\mathrm{CNOH}$ (Figure 6). Of these 649 were the Charnley and Exeter THR. The following groups of patients were excluded from the data analysis: 50 patients did not attend (DNA) the joint register, 9 patients we could not contact despite efforts (patient phone and next of kin phone not in use/GP unaware of patient location) and 10 patients opted-out of the joint register participation.

580 were either the Charnley (348) or the Exeter (232) THR. This included 67 patients who had died (44 Charnley/23 Exeter) since their last follow up and 14 patients who were in long term care, however their data was used and neither of the two groups were known to have any hip problems.

The Charnley and Exeter THRs had been performed by surgeons of varying experience including 16 orthopaedic surgical consultants who perform cemented THRs, three orthopaedic specialist registrars (SpRs) and four general orthopaedic registrars. All surgical procedures utilised third generation cementing techniques, and standard post-operative protocols were implemented in both groups.

\section{Demographics and PROS}

The pre-operative diagnosis was osteoarthritis in more than $90 \%$ of the cases for each implant (Table 1 ).

There were 348 cases of Charnley THR with a mean age of 68 years and BMI of 29.1 and in contrast there were 232 cases of Exeter THR with a mean age of 66.7 years and BMI of 28.7 at the time of surgery (Table 2).

Of the 348 Charnley cases (Table 3) 189 (54\%) were females with a mean age of 68.2 years (range of 18 - 90 years) at the time of surgery with a mean BMI of 28.2 (median 28.0) at a standard deviation of 4.894. The males were 159 (46\%) with a mean age of 68.1 (range of 38-86 years) at the time of surgery and a mean BMI 31.1 (median 30.0) at a standard deviation of 6.163. 


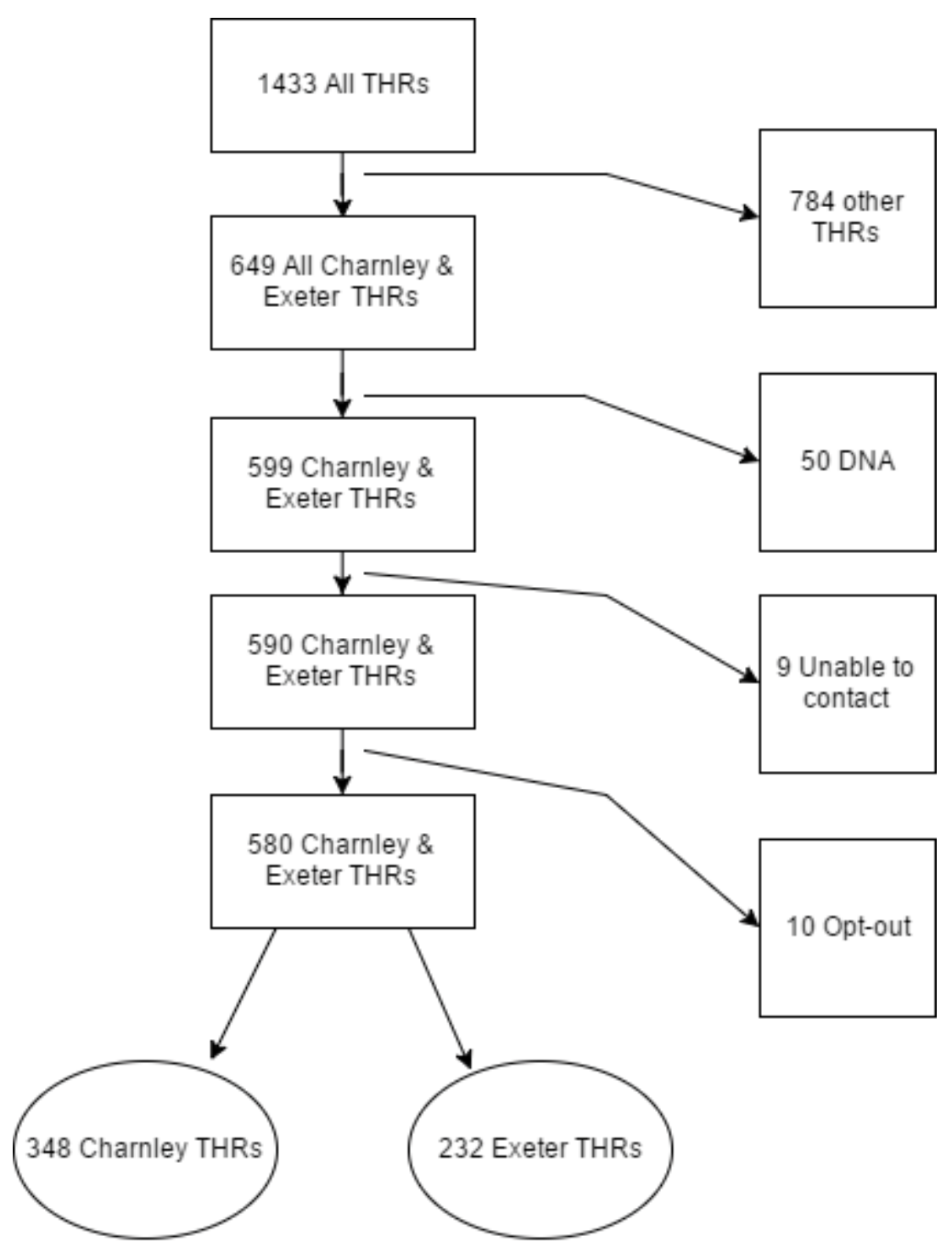

Figure 6: Charnley \& Exeter flow chart.

Table 1: Pre-operative diagnosis.

\begin{tabular}{|l|l|l|}
\hline Diagnosis & Charnley & Exeter \\
\hline Primary Osteoarthritis & $326(93.6 \%)$ & $211(90.9 \%)$ \\
\hline Rheumatoid Arthritis & $7(2 \%)$ & $3(1.2 \%)$ \\
\hline Post traumatic arthritis & $5(1.4 \%)$ & $3(1.2 \%)$ \\
\hline Developmental dysplasia of the hip & $4(1.1 \%)$ & $6(2.5 \%)$ \\
\hline Avascular necrosis & $4(1.1 \%)$ & $5(2.1 \%)$ \\
\hline Childhood SUFE (slipped upper femoral epiphysis) & $1(0.2 \%)$ & $2(0.86 \%)$ \\
\hline Perthes disease & $1(0.2 \%)$ & $1(0.43 \%$ \\
\hline Failed hemiarthroplasty & $0(0 \%)$ & $1(0.43 \%)$ \\
\hline
\end{tabular}

Table 2: Charnley \& Exeter THR demographics.

\begin{tabular}{|l|l|l|}
\hline & Charnley & Exeter \\
\hline Total number & 348 & 232 \\
\hline Mean age (years) & 68.1 & 66.7 \\
\hline Sex (male/female) & $159 / 189$ & $104 / 128$ \\
\hline Mean BMI & 29.1 & 28.7 \\
\hline
\end{tabular}

Table 3: Charnley THR demographics.

\begin{tabular}{|l|l|l|}
\hline Charnley THR & Male & Female \\
\hline Total number & $159(46 \%)$ & $189(54 \%)$ \\
\hline Mean age (years) & 68.1 & 68.2 \\
\hline Age range (years) & $38-86$ & $18-90$ \\
\hline Mean BMI & 31.1 & 28.2 \\
\hline
\end{tabular}




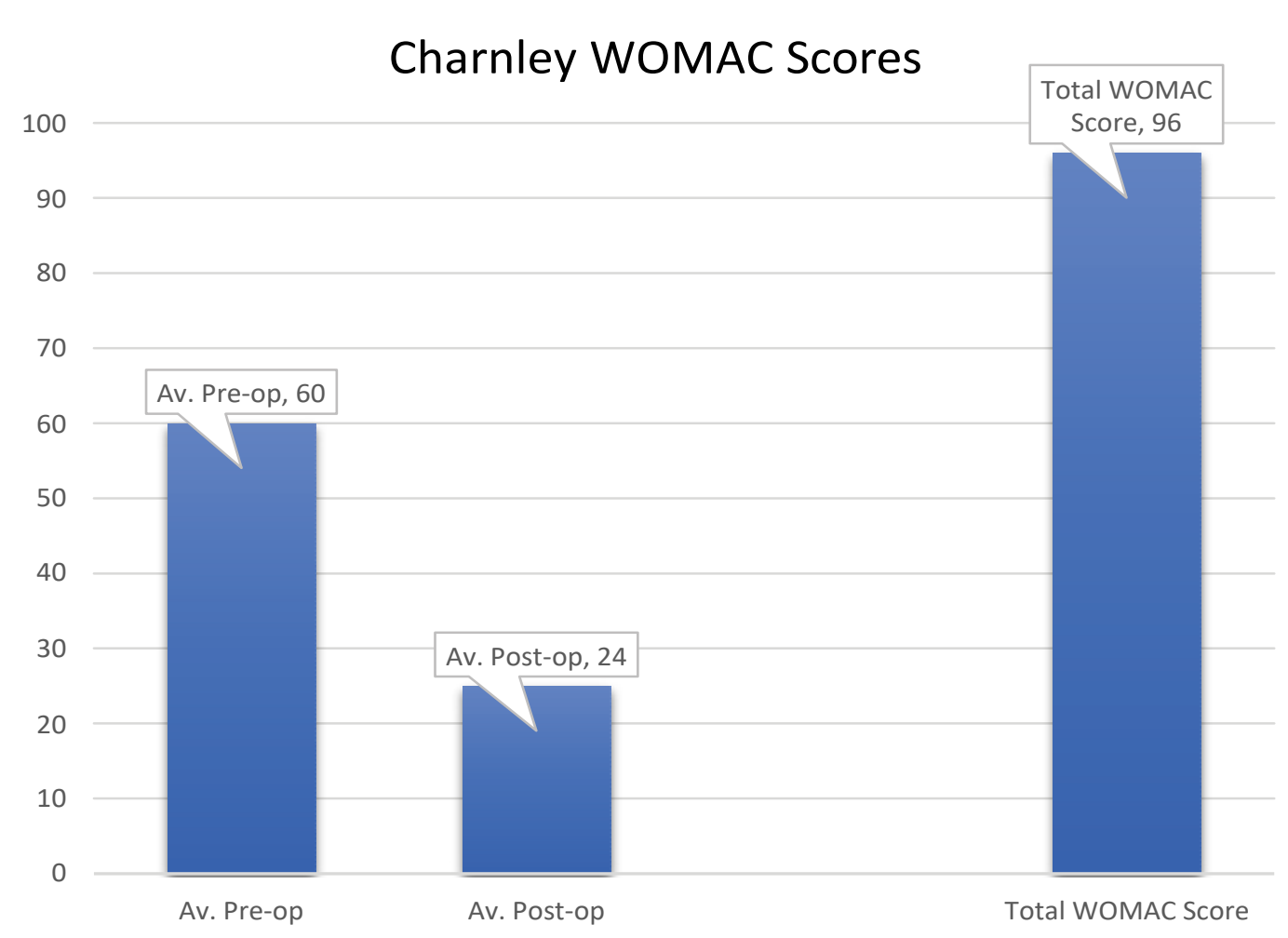

Figure 7: Charnley WOMAC scores.

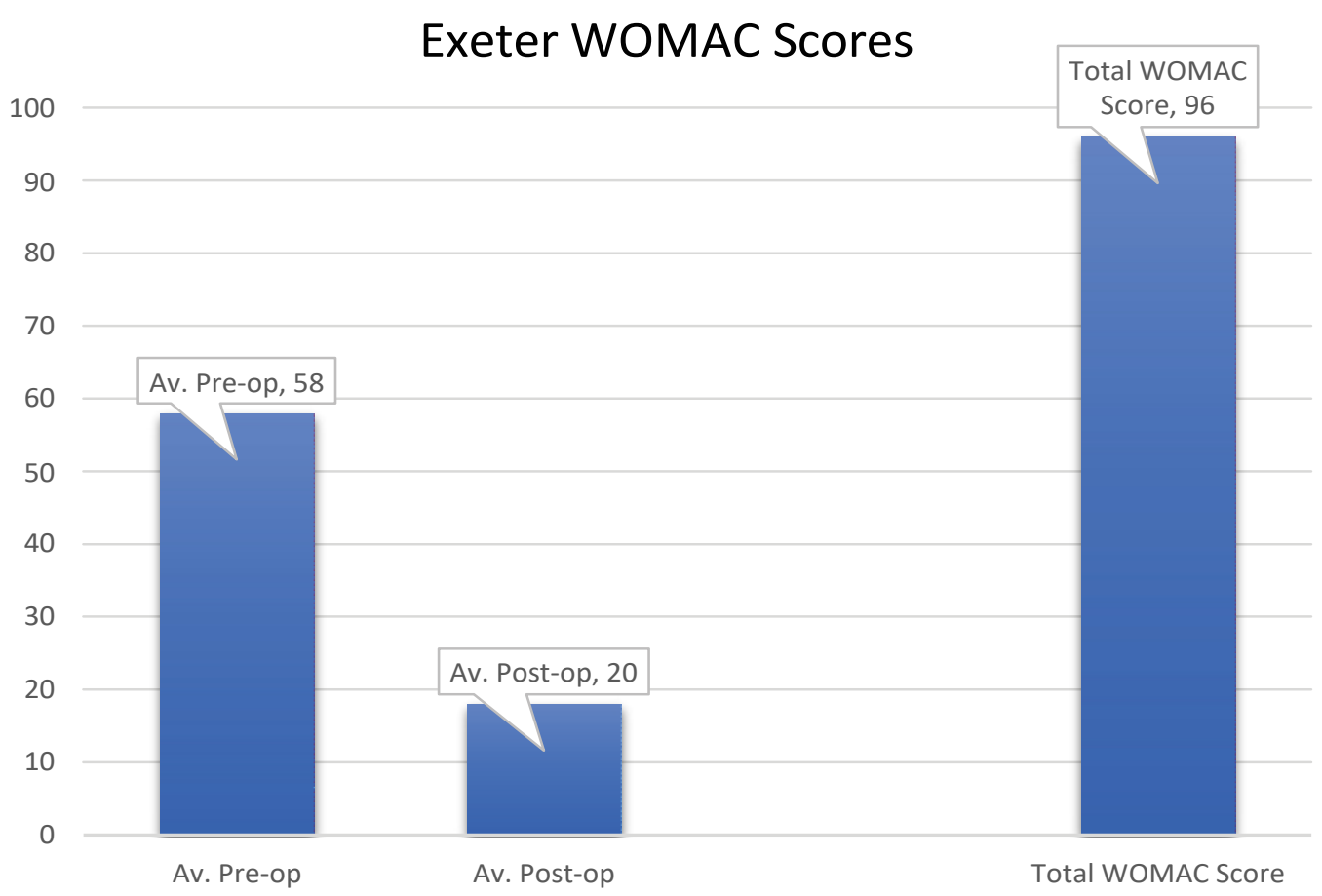

Figure 8: Exeter WOMAC scores.

Table 4: Exeter THR demographics.

\begin{tabular}{|l|l|l|}
\hline Exeter THR & Male & Female \\
\hline Total number & $104(45 \%)$ & $128(55 \%)$ \\
\hline Mean age (years) & 65.1 & 68.1 \\
\hline Age range (years) & $25-85$ & $38-84$ \\
\hline Mean BMI & 28.7 & 28.8 \\
\hline
\end{tabular}

In the Exeter group, there were 232 patients (Table 4) of which 128 (55\%) were females with a mean age of 68.1 years (range of 38-84 years) and a mean BMI of 28.76 (median 29.0) at a standard deviation of 5.8. The males were 104 (45\%) with a mean age of 65.1 years (range of 25-85 years) and a mean BMI of 28.71 (median 27.5) standard deviation of 5.55 at the time of surgery.

The average pre-operative WOMAC scores in the 
Charnley group was 60 and had reduced to 24 at the 10 year review. The average pre-operative WOMAC scores in the Exeter group was 58 and had reduced to 20 at the 10 years review (Figure 7 and Figure 8).

There was no significant difference in WOMAC scores between the two groups at ten years $(p=0.08$ Mann-Whitney U).

The different types of acetabular cups used in the Charnley group were 344 Ogee cups, three Elite plus cups and one Contemporary cup. Meanwhile the different types of acetabular cups used in the Exeter group were 150 Contemporary cups, 22 Elite plus cups, 57 Trident cups and three Ogee cups (Figure 9 and Figure 10).
Regression analysis was performed to assess if patients age predicted which stem they would get implanted (Table 5). R-squared $=0.004(0.4 \%)$ and adjusted R-square $=0.002(0.2 \%)$ indicating that there is almost no chance that the THR type used was affected by the age of the patient. The ANOVA test $F$ value was at $2.098(p=0.148)$ which was not statistically significant, confirming our results that THR type used on patients was not related to the patients age and rather due to other factors such as the surgeons experience and training.

\section{Survival analysis}

The Charnley group had seven revisions and the Exeter group had two revivions. Two of the revisions

\section{Charnley acetabular cups}

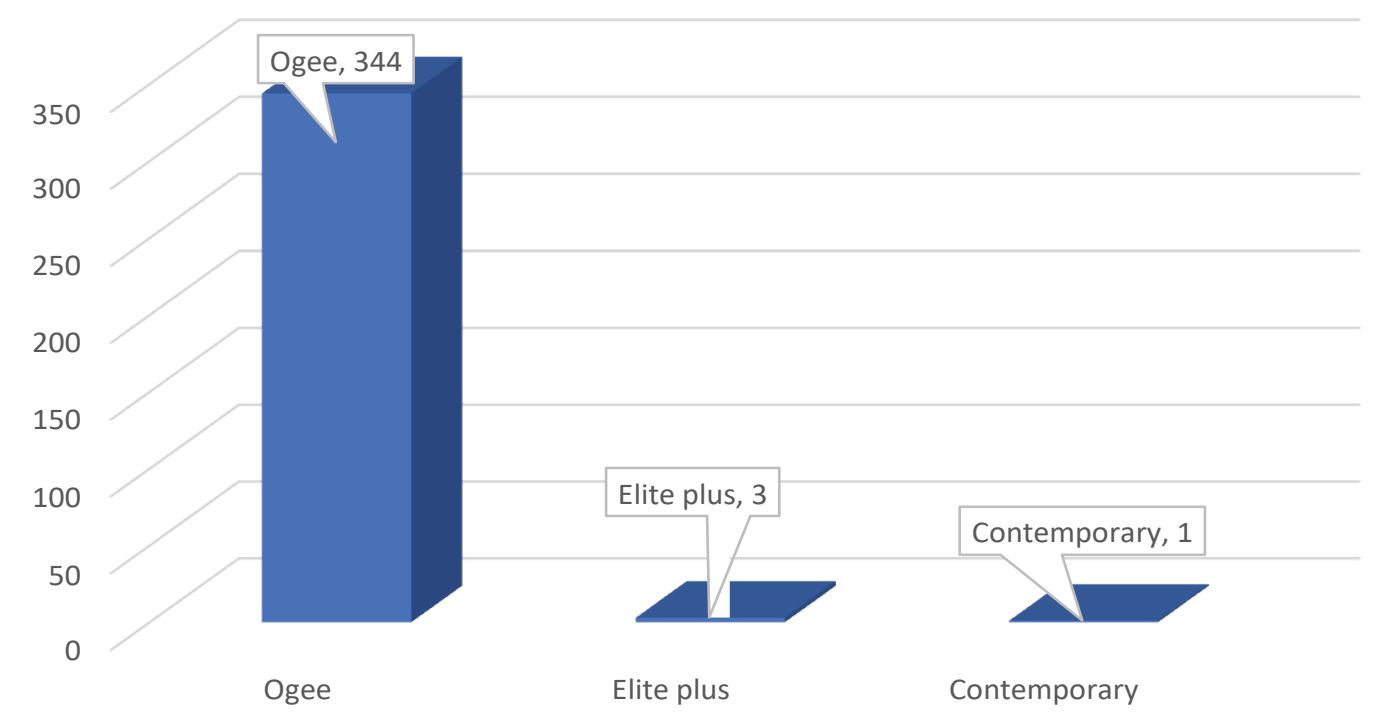

Figure 9: Charnley acetabular cup.

\section{Exeter acetabular cups}

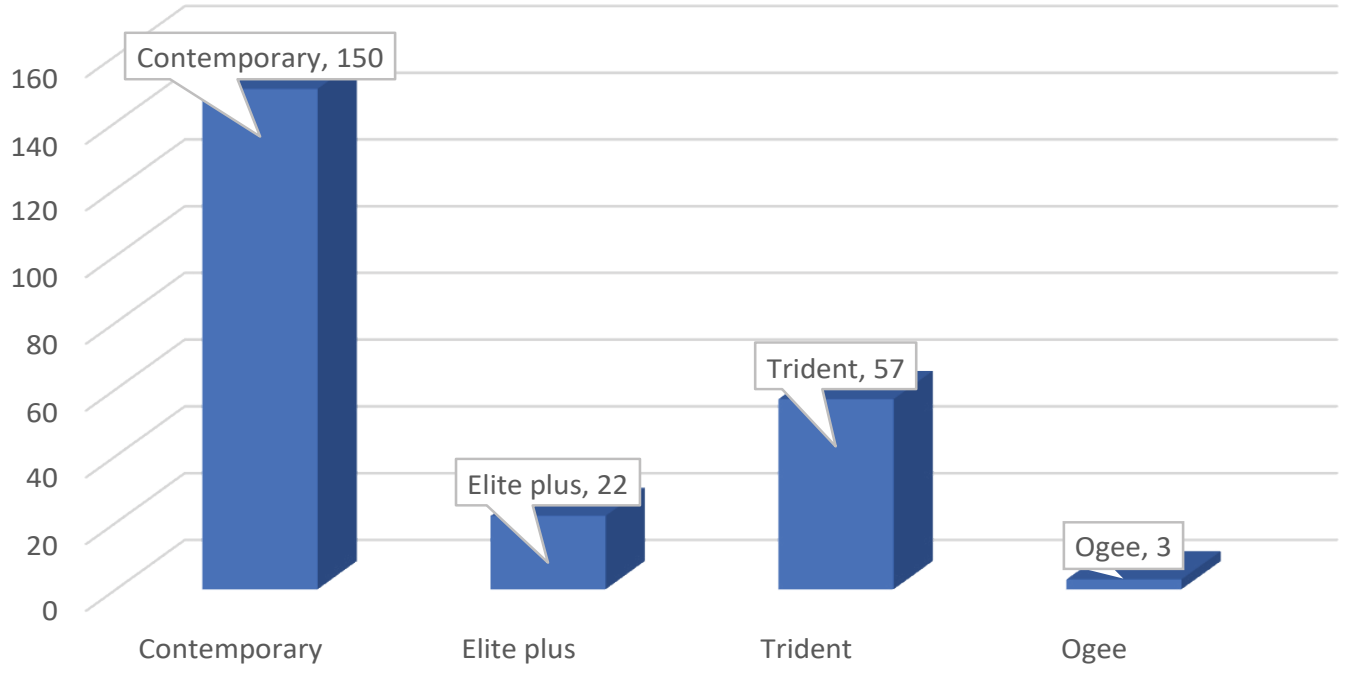

Figure 10: Exeter acetabular cups. 
Table 5: Regression outputs for age with stem (model summary and ANOVA test).

\begin{tabular}{|l|l|l|l|l|}
\hline \multicolumn{5}{|c|}{ Model Summary } \\
\hline Model & R & R Square & Adjusted R Square & Std. Error of the Estimate \\
\hline 1 & $0.060^{\mathrm{a}}$ & 0.004 & 0.002 & 0.490 \\
\hline
\end{tabular}

aPredictors: (Constant), AGE_DOS

\begin{tabular}{|c|c|c|c|c|c|c|}
\hline \multicolumn{7}{|c|}{ ANOVA $^{a}$} \\
\hline \multirow{2}{*}{\begin{tabular}{|l} 
Model \\
1
\end{tabular}} & \multicolumn{2}{|c|}{ Sum of Squares } & \multirow{2}{*}{$\begin{array}{l}\text { df } \\
1\end{array}$} & \multirow{2}{*}{$\begin{array}{l}\text { Mean Square } \\
0.503\end{array}$} & \multirow{2}{*}{\begin{tabular}{|l|}
$F$ \\
2.098
\end{tabular}} & \multirow{2}{*}{$\begin{array}{l}\text { Sig } \\
0.148^{b}\end{array}$} \\
\hline & Regression & 0.503 & & & & \\
\hline & Residual & 138.336 & 579 & 0.240 & & \\
\hline & Total & 138.839 & 580 & & & \\
\hline
\end{tabular}

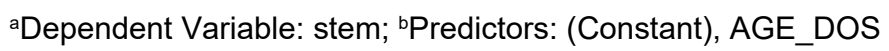

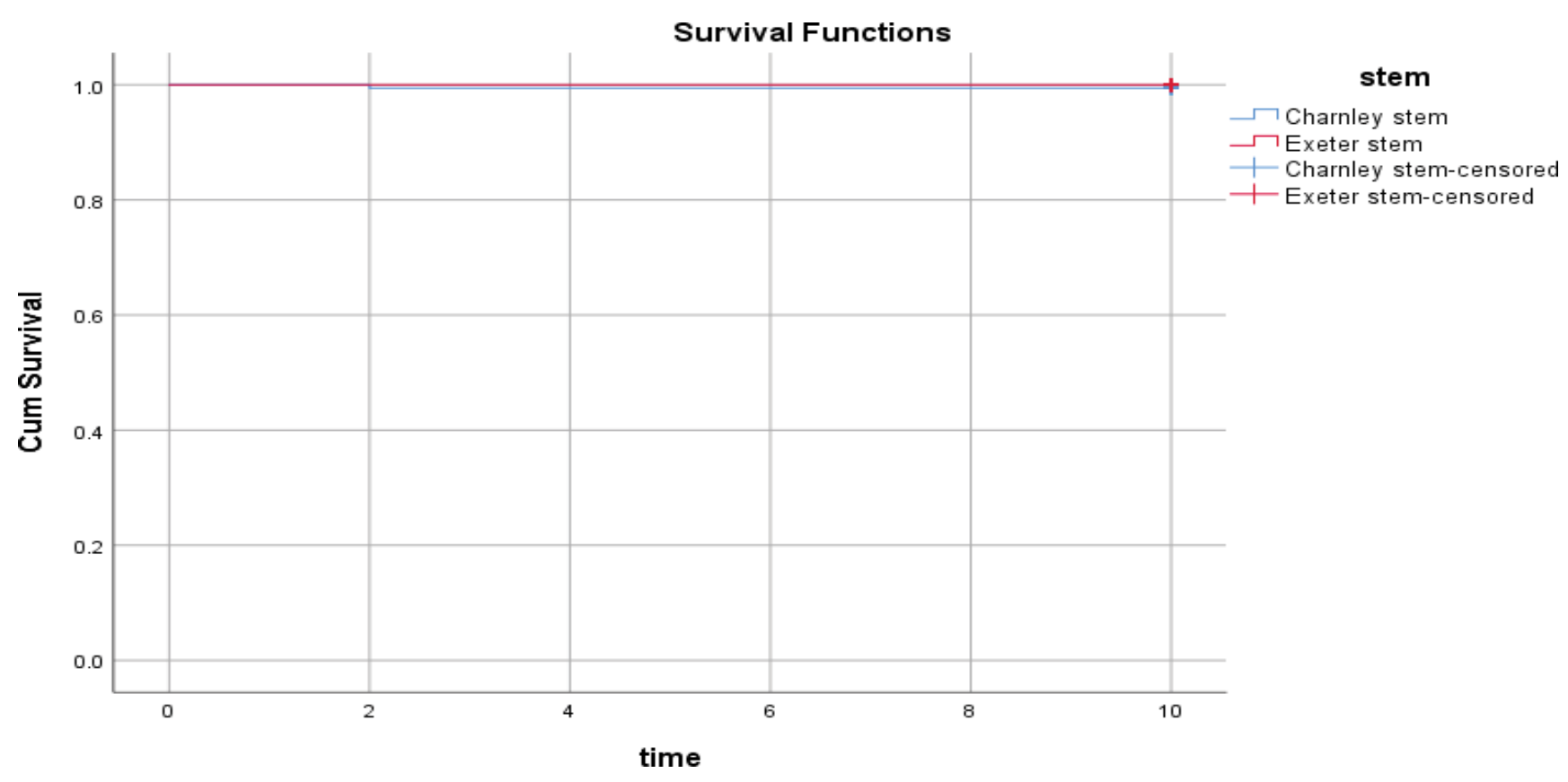

Figure 11: Kaplan Meier survival graph of stems taking aseptic loosening as the end point.

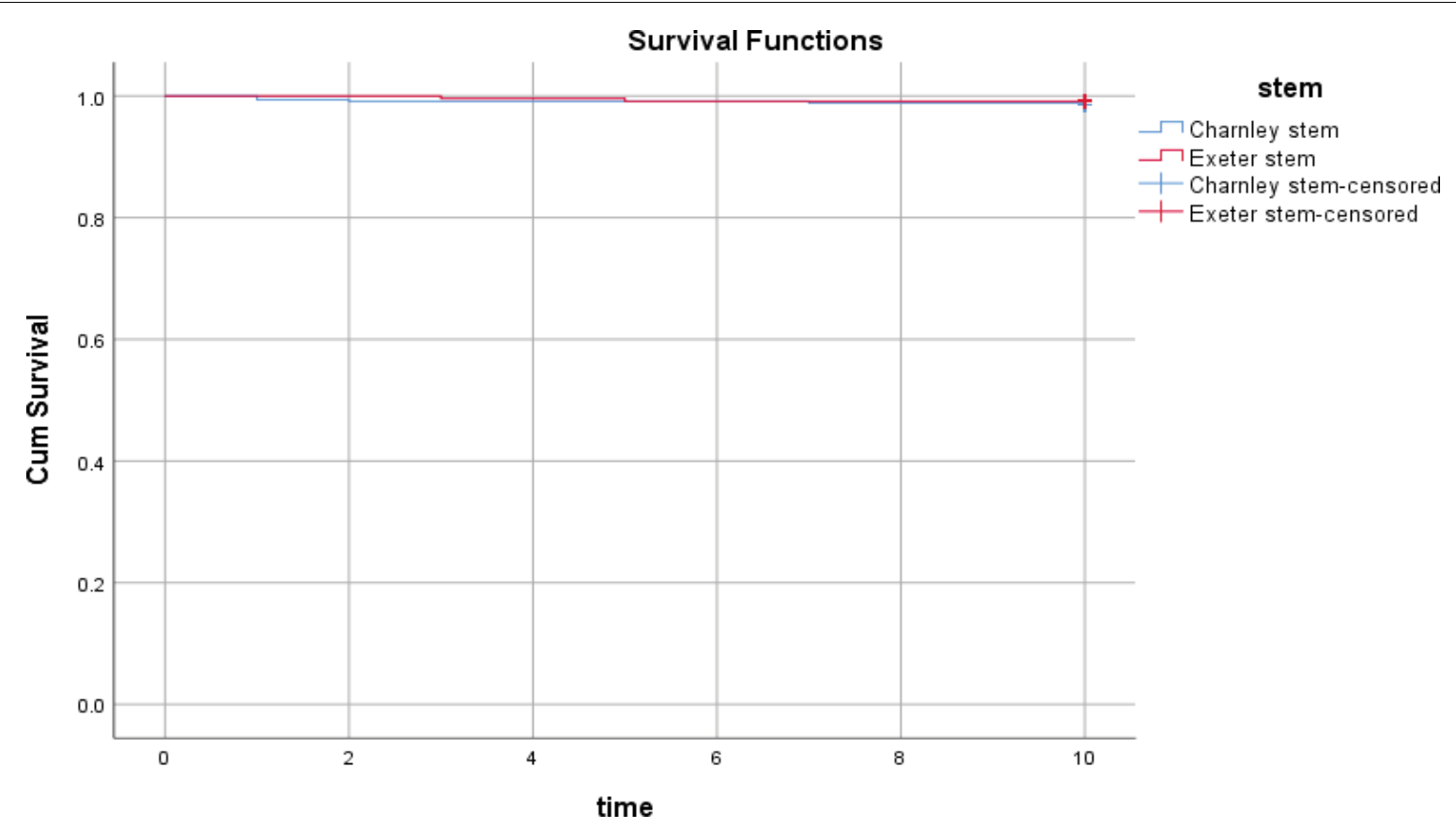

Figure 12: Kaplan Meier survival graph of stems taking revision for other causes. 
in the Charnley group were for aseptic loosening of the stem. Thus, there was a $99.4 \%$ survivorship in the Charnley group. The combination was a Charnley stem and Ogee cup for both revisions.

There were no revisions for aseptic loosening of the stem in the Exeter group and therefore the Exeter THRs had a $100 \%$ survivorship (Figure 11).

There was no significant difference between the stems survival for aseptic loosening at ten years $(p=$ 0.249 Log Rank test of sensitivity).

Taking Charnley revisions for other causes as the end point, there were three deep infections requiring revision, all of the Charnley stem and Ogee cup combination. There were also two recurrent dislocations which required revisions, both of the Charnley and Ogee cup resulting in a survivorship of $98.5 \%$ for other reasons.

In the Exeter group there was one revision for a deep infection which was an Exeter stem and Contemporary cup. One revision for a periprosthetic fracture around a well-fixed implant which was an Exeter stem and Trident cup following a fall. This resulted in a survivorship of 99.1\% for other causes of revision (Figure 12).

There was no significant difference between the stems survival for other causes of revision ( $p=0.533 \mathrm{Log}$ Rank test of sensitivity).

\section{Discussion}

\section{Principle findings}

Survivorship for any of the components for aseptic loosening was $99.4 \%$ vs. $100 \%$ for the Charnley and Exeter THRs respectively. Survivorship of the Charnley and Exeter THR for other causes was $98.5 \%$ vs. $99.1 \%$. The overall survivorship for the Charnley THR was $97.9 \%$ compared to $99.1 \%$ in the Exeter THR group.

The combination of stem and implant was quite standard for the Charnley THRs with the standard Ogee cup being used in $98.8 \%$ (344 cups) of the cases, Elite plus cups used three times and one contemporary cup implanted, all of which are cemented acetabular components.

However in the Exeter group there was a mixture of acetabular components used in addition to the standard cemented Contemporary cup being implanted only $64 \%$ (150 cups) of the time. The use of the uncemented Trident cup was used almost a quarter of the time (24.5\%) (57 cups) resulting in a hybrid THR with no loosening and only one revision due to a periprosthetic fracture around a well fixed implant. The use of the hybrid combination is usually reserved for the younger patients with good acetabular bone stock. In our cohort the patients who received the Tridnet cup were 65 years of age or younger. The Elite plus cup which its counterpart is usually the Elite modular Charnley stem was used $9 \%$ ( 22 cups) of the time. The Ogee acetabular cups were used three times (1\%).

There were 18 short Exeter stems used, 15 in females and three in males. All the short Exeter stems had good outcome and survivorship throughout this study.

When comparing patient reported outcome scores through the WOMAC scores we can see that the pain symptoms markedly improved in both groups, but the Exeter group showed better results than the Charnley group reflected by the drop in scores from 58 to 20 in the Exeter THRs and from 60 to 24 in the Charnley THRs. However the difference in results was not proven to be statistically significant. Unfortunately what was noticed during the study period is that the WOMAC scores can be misleading and they are more sensitive than specific, meaning that patients with other problems/OA in other body parts specifically the back tend to have worse scores than patients who have good backs irrespective of the THR outcome.

Our results with the Charnley THR have shown similar and improved survivorship results compared to other 10-year studies [34,38,39], nevertheless these studies had the potential to prove low revision rates beyond 20 years due to their long follow-up period which we cannot compare to yet. However, results with the Exeter stem confirm those of other published studies $[22,28,40]$.

\section{Strengths}

We are not aware of any published Irish studies relating to survivorship of THRs and patient reported outcomes from joint registries, $\mathrm{CNOH}$ joint register is the longest active hospital based joint register in Ireland and recently passed the ten-year mark.

Many factors affect the outcome of cemented THR; the expertise and skills of the orthopaedic surgeon, the cementation method and technique as well as patient factors. In $\mathrm{CNOH}$ the THRs in both groups were performed by consultant orthopaedic surgeons and trainees at varying levels, however all the trainees had been taught the procedure by their consultant orthopaedic surgeon and performed unsupervised surgery only after they were considered to be competent in carrying out THRs. We consider that to have made our sample quite homogenous and added strength to our study results.

\section{Limitations}

Our limitations with this study is that we had not correlated the radiographic results with the revised hips and whether there was any marked loosening in the other hips that had not been revised. We appreciate the fact that the electronic radiographic system does not integrate with the joint register system and looking at each X-Ray individually may be time consuming due to the large number of THRs taking place; it would be efficient if the X-Ray reports could be uploaded onto 
the joint register to make studies easier in correlating radiographic results. This may be undertaken in a future study from $\mathrm{CNOH}$.

Looking at our patient reported outcomes through the WOMAC scores may not be ideal individually as mentioned earlier although the form is quite informative, however using a joint assessment specific form questionnaire in addition to the WOMAC may reflect the true patient reported outcomes after THR.

A total of 69 patients had been excluded from this study (50 DNA - 9 unable to contact - 10 Opt-out) which could have affected our results if this study was a multicenter research as the numbers excluded would have been higher. Attempting to locate these patients and include their data in the study would increase the strength of this research as we would be able to account for all procedures and outcomes of the THRs performed in $\mathrm{CNOH}$.

\section{Conclusion}

From this study our results of the Charnley and Exeter THR in terms of survivorship and outcomes are excellent and following NICE guidelines with respect to revision rates.

We shall continue to follow-up these patients and would recommend a review of the survivorship and outcomes of these THRs at the 15 and also at the 20year mark to compare results with other joint registries and to assess whether $\mathrm{CNOH}$ revision rates are in line with international standards and NICE guidelines as they are at the 10-year mark.

\section{References}

1. (2017) Osteoarthritis: Care and management. National Institue for Health and Care Excellence.

2. Woolf AD, Pfleger B (2003) Burden of major musculoskeletal conditions. Bulletin of the World Health Organization. 81: 646-656.

3. Glyn-Jones S, Palmer AJR, Agricola R, Price AJ, Vincent TL, et al. (9991) Osteoarthritis. The Lancet 386: 376-387.

4. http://clinics.irishhealth.com/rheumatoid-arthritis/basics/ what-is-osteoarthritis/

5. French HP, Galvin R, Horgan NF, Kenny RA (2016) Prevalence and burden of osteoarthritis amongst older people in Ireland: Findings from The Irish LongituDinal Study on Ageing (TILDA). European Journal of Public Health 26: 192-198.

6. Tubach F, Ravaud P, Baron G, Falissard B, Logeart I, et al (2005) Evaluation of clinically relevant changes in patient reported outcomes in knee and hip osteoarthritis: The minimal clinically important improvement. Ann Rheum Dis 64: 29-33.

7. Nyfos $L$ (1983) Traction therapy of osteoarthrosis of the hip. A controlled study. Ugeskrift laeger 145: 2837-2840.

8. Klassbo M, Larsson G, Harms-Ringdahl K (2003) Promising outcome of a hip school for patients with hip dysfunction. Arthritis Rheum 49: 321-327.
9. Fernandes L, Storheim K, Sandvik L, Nordsletten L, Risberg MA (2010) Efficacy of patient education and supervised exercise vs patient education alone in patients with hip osteoarthritis: A single blind randomized clinical trial. Osteoarthritis Cartilage 18: 1237-1243.

10. Hunter DJ, Lo GH (2009) The management of osteoarthritis: An overview and call to appropriate conservative treatment. Rheumatic Disease Clinics of North America 34: 689-712.

11. Pateder DB, Hungerford MW (2007) Use of fluoroscopically guided intra-articular hip injection in differentiating the pain source in concomitant hip and lumbar spine arthritis. Am J Orthop (Belle Mead NJ) 36: 591-593.

12. Singh JA (2011) Epidemiology of knee and hip arthroplasty: A systematic review. Open Orthop J 5: 80-85.

13. Rachel Wittenauer LS, Kamal Aden. Osteoarthritis. WHO, 2013 Contract No.: Background Paper 6.12.

14. Hunter DJ, Felson DT (2006) Osteoarthritis. BMJ 332: 639642.

15. Pivec R, Johnson AJ, Mears SC, Mont MA (2012) Hip arthroplasty. The Lancet 380: 1768-1777.

16. Mellon SJ, Liddle AD, Pandit $H$ (2013) Hip replacement: Landmark surgery in modern medical history. Maturitas 75 : 221-226.

17. Grammatopoulos G, Pandit H, Glyn-Jones S, McLardySmith P, Gundle R, et al. (2010) Optimal acetabular orientation for hip resurfacing. J Bone Joint Surg Br 92: 1072-1088.

18. (2017) Health Service Executive. Hip replacement.

19. Kandala N-B, Connock M, Pulikottil-Jacob R, Sutcliffe P, Crowther MJ, et al. (2015) Setting benchmark revision rates for total hip replacement: Analysis of registry evidence. BMJ: British Medical Journal 350: h756.

20. Charnley J (1961) Arthroplasty of the hip. A new operation. Lancet 1: 1129-1132.

21. Mirza SB (2010) Basic science considerations in primary total hip replacement arthroplasty. Open Orthop J 4: 169-180.

22. (2016) National Joint Registry. 13th Annual Report 2016 - National Joint Registry for England, Wales, Northern Ireland and the Isle of Man 2016.

23. (2005) Healio - orthopaedics. Evolution of Cemented Stems.

24. Knight SR (2011) Total Hip Arthroplasty - over 100 years of operative history. Orthopaedic Reviews 3.

25. (2007) Core Knowledge in Orthopaedics-Pediatric Orthopaedics. Journal of Pediatric Orthopaedics 27: 602.

26. Havelin LI, Engesaeter LB, Espehaug B, Furnes O, Lie SA, et al. (2000) The norwegian arthroplasty register: 11 years and 73,000 arthroplasties. Acta Orthop Scand 71: 337-353.

27. Nercessian OA, Martin G, Joshi RP, Su BW, Eftekhar NS (2005) A 15- to 25- Year follow-up study of primary charnley low-friction arthroplasty: A single surgeon series. J Arthroplasty 20: 162-167.

28. Sherfey JJ, McCalden RW (2006) Mid-term results of Exeter vs Endurance cemented stems. J Arthroplasty 21: 1118-1123.

29. National Health Service - NHS Supply Chain. ODEP criteria.

30. Srinivasan A, Jung E, Levine BR (2012) Modularity of the femoral component in total hip arthroplasty. J Am Acad Orthop Surg 20: 214-222. 
31. Poon PC, Rennie J, Gray DH (2001) Review of total hip replacement. The Middlemore Hospital experience, 19801991. N Z Med J 114: 254-256.

32. Klapach AS, Callaghan JJ, Goetz DD, Olejniczak JP, Johnston RC (2001) Charnley total hip arthroplasty with use of improved cementing techniques: A minimum twentyyear follow-up study. J Bone Joint Surg Am 83: 1840-1848.

33. Soyer J, Avedikian J, Pries P, Clarac JP (1997) Long-term outcome of Charnley's femoral implant. A review of 309 cases with follow-up of minimum 20 years. Rev Chir Orthop Reparatrice Appar Mot 83: 416-422.

34. Mullins MM, Norbury W, Dowell JK, Heywood-Waddington M (2007) Thirty-year results of a prospective study of Charnley total hip arthroplasty by the posterior approach. The Journal of Arthroplasty 22: 833-839.

35. Caton J, Prudhon JL (2011) 25 years survival after Charnley's total hip arthroplasty. Int Orthop 35: 185-188.

36. Berry DJ, Harmsen WS, Cabanela ME, Morrey BF (2002) Twenty-five-year survivorship of two thousand consecutive primary Charnley total hip replacements: Factors affecting survivorship of acetabular and femoral components. J Bone Joint Surg Am 84: 171-177.

37. Espehaug B, Furnes O, Engesæter LB, Havelin LI (2009) 18 years of results with cemented primary hip prostheses in the Norwegian Arthroplasty Register. Acta Orthopaedica 80: $402-412$

38. Junnila M, Laaksonen I, Eskelinen A, Pulkkinen P, Ivar Havelin L, et al. (2016) Implant survival of the most common cemented total hip devices from the Nordic Arthroplasty Register Association database. Acta Orthopaedica 87: 546553.

39. (2016) Australian Orthopaedic Association. Australian Orthopaedic Association - National Joint Replacement Registry.

40. Williams HD, Browne G, Gie GA, Ling RS, Timperley AJ, et al. (2002) The Exeter universal cemented femoral component at 8 to 12 years. A study of the first 325 hips. J Bone Joint Surg Br 84: 324-334.

41. (2013) National Joint Registry. 10th Annual Report 2013 - National Joint Registry for England, Wales and Northern Ireland.

42. Franklin J, Robertsson O, Gestsson J, Lohmander LS, Ingvarsson T (2003) Revision and complication rates in 654 Exeter total hip replacements, with a maximum follow-up of 20 years. BMC Musculoskeletal Disorders 4: 6.

43. Carrington NC, Sierra RJ, Gie GA, Hubble MJ, Timperley AJ, et al. (2009) The Exeter Universal cemented femoral component at 15 to 17 years: An update on the first 325 hips. J Bone Joint Surg Br 91: 730-737.

44. Petheram TG, Whitehouse SL, Kazi HA, Hubble MJ, Timperley AJ, et al. (2016) The Exeter Universal cemented femoral stem at 20 to 25 years: A report of 382 hips. Bone Joint J 98: 1441-1449.
45. Schmitz MW, Bronsema E, de Kam DC, Gardeniers JW, Veth RP, et al. (2017) Results of the cemented Exeter femoral component in patients under the age of 40: An update at ten to 20 years' follow-up. Bone Joint J 99: 192198.

46. Ulrich SD, Seyler TM, Bennett D, Delanois RE, Saleh KJ, et al. (2008) Total hip arthroplasties: What are the reasons for revision? Int Orthop 32: 597-604.

47. Prokopetz JJ, Losina E, Bliss RL, Wright J, Baron JA, et al. (2012) Risk factors for revision of primary total hip arthroplasty: a systematic review. BMC Musculoskeletal Disorders 13: 251.

48. National Joint Registry. NJR National Joint Registry England and Wales 9th Annual Report 2012. Healthcare Quality Improvement Partnership: 2012.

49. Keurentjes JC, Pijls BG, Van Tol FR, Mentink JF, Mes SD, et al. (2014) Which implant should we use for primary total hip replacement? A systematic review and meta-analysis. The Journal of Bone and Joint Surgery American volume 1: 79-97.

50. http://www.njrcentre.org.uk/njrcentre/default.aspx

51. Elmallah RK, Krebs VE, Mont MA (2015) National and hospital registries: An invaluable source and wealth of information. J Arthroplasty 30: 1673-1675.

52. Maloney WJ (2001) National Joint Replacement Registries: Has the time come? J Bone Joint Surg Am 83: 1582-1585.

53. Smith AJ, Dieppe P, Vernon K, Porter M, Blom AW (2012) Failure rates of stemmed metal-on-metal hip replacements: Analysis of data from the national joint registry of england and wales. Lancet 379: 1199-1204.

54. Kurtz S, Mowat F, Ong K, Chan N, Lau E, et al. (2005) Prevalence of primary and revision total hip and knee arthroplasty in the United States from 1990 through 2002. J Bone Joint Surg Am 87: 1487-1497.

55. Herberts $P$, Malchau $H(2000)$ Long-term registration has improved the quality of hip replacement: A review of the Swedish THR Register comparing 160,000 cases. Acta Orthopedica Scandinavica 71: 111-121.

56. Pachore JA, Vaidya SV, Thakkar CJ, Bhalodia HKP, Wakankar HM (2013) ISHKS joint registry: A preliminary report. Indian J Orthop 47: 505-509.

57. National Office of Clinical Audit NOCA. INOR Methodology.

58. O'Neill BJ, Nugent M, Cashman JP, O'Flanagan SJ, Keogh P, Kenny PJ (2014) The irish national joint registry: Where are we now? Ir J Med Sci 183: 77-83.

59. (2015) Cappagh national orthopaedic hospital annual report 2015.

60. McGrory BJ, Harris WH (1996) Can the western Ontario and McMaster Universities (WOMAC) osteoarthritis index be used to evaluate different hip joints in the same patient? The Journal of Arthroplasty 11: 841-844. 